\title{
MICROSCOPIC ALGAE AND PROTOZOA IN THE WATERS NEAR PLYMOUTH IN AUGUST 1962
}

\author{
By James B. Lackey and Elsie W. LACKey \\ University of Florida, Gainesville, Florida
}

Larger algae and protozoa plankton of inshore waters seem generally well known, except for a few groups of very small flagellates. The same statement cannot be made for the micro-organisms of the bottom sediment-water interfaces and intertidal sand. Many ecologists and physiologists would like to know more about what species and how many individuals can be expected in such locations as these.

\section{QUALITATIVE RESULTS}

In August 1962, while investigating the colourless euglenids of the Plymouth area, we listed all microscopic algae and protozoa which could be identified, as shown in Table I. This list is not exhaustive; it omits many organisms which could not be identified because too few were seen, or which could not be stilled, or because there was not enough time to run them down. Plankton diatoms are easy to identify, but bottom-dwelling ones demanded more time than was available. Hence few of them are included. Rhizopods and bluegreen algae were omitted from the list for the same reason.

Material examined came from the following sources: (I) surface townettings inside and beyond the breakwater with 600 m.p.i. nets, and centrifuged catches from the same locations; (2) organisms in the sand between tide marks on Drake's Island and near the laboratory; (3) sediment from the bottom of the Tamar River to a point several miles upstream, and sediment from the mud banks of the River Plym at low tide.

Sand was sampled by digging until water flowed into the hole, the water was then violently agitated, and a dip sample taken. Sediment was collected by the use of a grab sampler, or directly by hand. Material was brought into the laboratory in small containers and kept $\operatorname{cool}\left(18^{\circ} \mathrm{C}\right.$ or thereabouts). Some material from each sample was examined at once, some was allowed to stand, and Petri dish moist chambers were used to keep slides overnight, giving many organisms a chance to become quiescent.

Winchester bottles were filled, kept cool, and on reaching the laboratory, $60 \mathrm{ml}$. portions of the water were centrifuged at 2200 r.p.m. in conical-ended tubes for 5 minutes, then the plankton was identified. 


\section{ORGANISMS FOUND}

Table I is a list of the organisms identified in the Ioo or more samples examined. The current fauna list (Marine Biological Assoc., I957) cites 89 ciliates and Io zooflagellates. Table I lists 252 genera or species. Since none of the groups shown in Table I are comprehensive, this is a further indication of the richness of the Plymouth biota. This is especially true of the mud flats of the Plym estuary for sulphur bacteria and colourless euglenids, and of the region beyond the breakwater for chrysomonads. Only I3 ciliates from the fauna list are included in Table I, so that at least I57 ciliates have been found at Plymouth.

\section{TABLE 1. MICRO-ORGANISMS OF THE PLYMOUTH AREA}

Column I, plankton beyond the breakwater; column 2, plankton inside the breakwater; column 3 , organisms in the sand and mud flats; column 4, organisms in the sediment.

I. BACTERIA-8

I

Beggiatoa alba (Vaucher) Trevisan

$B$. arachnoidea (Agardh) Rabenhorst

$B$. gigantea Klas

B. leptomitiformis Trevisan

B. minima Winogradsky

B. mirabilis Cohn

Thiothrix nivea (Rabenhorst) Winogradsky

Thiovulum majus Hinze

2. EUglenophyta-28

Anisonema sp. A

A. emarginatum Stokes

Anisonema sp. B

$A$. ovale Klebs

A. pusillum Stokes

Dinema grisoleum Perty

D. litorale Skuja

Entosiphon sp.

Euglena fenestrata Elenkin

$E$. vermiformis $\mathrm{N}$. Carter

Eutreptia colourless sp.

E. lanowii Steuer

E. viridis Perty

Heteronema acus (Ehrenb.) Stein

Metanema variabile Klebs

Notosolenus apocamptus Stokes

Pentamonas spinifera Lackey

Peranema cuneatum Playfair

P. trichophorum (Ehrenb.) Stein

Petalomonas excavata Skuja

$P$. mediocanellata Stein

$P$. pusilla Skuja

Scytomonas pusilla Stein

Sphenomonas elongata Lackey

S. quadrangularis Stein

Tropidocyphus octocostatus Stein

Urceolus cyclostomus (Stein) Mereschowski

U. pascheri Skvortzow

U. sabulosus Stokes 
TABLE 1 (cont.)

3. ChLorophyceae-II

Chlamydomonas spp.

Collodictyon $\mathrm{sp}$.?

C. triciliatum Carter

Bipedinomonas rotunda $\mathrm{N}$. Carter

Pyramimonas grossii Parke

$P$. plurioculata Butch.

$P$. octociliata N. Carter

Thalassomonas minima Butch.

T. semiglobata Butch.

Halosphaera viridis Schmitz

Nannochloris bacillaris Naumann

4. CHRysophycEAe-6

Chrysococcus cinctus Lackey

Chrysochromulina strobilus Parke \&

Manton

Chrysochromulina spp.

Kephyrion ovum Pascher

Olisthodiscus luteus N. Carter

Pedinella hexacostata Wyssotski

Pseudopedinella pyriformis N. Carter

Chrysophyceae, unid.

5. CRYPTOPHYCEAe-5

Chilomonas marina (Braarud) Halldal

Chroomonas spp.

Cryptomonas spp.

Cyathomonas truncata (Ehrenb.) Fromentel . Rhodomonas spp.

6. Chloromonadophyceae-2

Thaumatomastix $\mathrm{sp}$.

$T$. setifera Lauterborn

7. CocCOLITHOPHORA-2

Cricosphaera carterae (Braarud \&

Fagerl.) Braarud

Pontosphaera sp.

$P$. huxleyi Lohmann

Syracosphaera sp.

8. Silicoflagellata-2

Dictyocha fibula Ehrenb.

Distephanus speculum Ehrenb.

9. Dinophyceae-49

Amphidinium bipes Herdman

A. ovum Herdman

A. scissoides Lebour

A. sulcatum Kofoid

A. testudo Herdman

A. vigrense Woloszynska

$A$. vitreum Herdman

Ceratium furca (Ehrenb.) Clap. \&

Lachm.

C. longipes (Bailey) Gran

C. minutum Jörgensen

C. tripos (Müller) Nitzsch

Dinophysis ovum Schütt

$D$. tripos Gourret

Diplopsalis lenticula Bergh

Diplopsalopsis orbicularis (Pauls.)

Meunier

\begin{tabular}{|c|c|c|}
\hline $\mathbf{I}$ & 2 & 3 \\
\hline$x$ & & \\
\hline$x$ & $\times$ & \\
\hline$x$ & . & . \\
\hline $\begin{array}{l}x \\
x\end{array}$ & $\dot{x}$ & $\cdot$ \\
\hline$\hat{x}$ & & \\
\hline & $\times$ & \\
\hline$\times$ & $\times$ & . \\
\hline$\dot{x}$ & $\dot{x}$ & 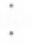 \\
\hline$x$ & & \\
\hline$\times$ & $\times$ & \\
\hline$\times$ & & \\
\hline$\times$ & . & . \\
\hline$\times$ & $\times$ & . \\
\hline$x$ & & . \\
\hline$x$ & & . \\
\hline $\begin{array}{l}x \\
x\end{array}$ & &. \\
\hline$x$ & $\times$ & . \\
\hline$\times$ & $\times$ & \\
\hline & . & $\begin{array}{l}x \\
x\end{array}$ \\
\hline$\dot{x}$ & $\therefore$ & $\begin{array}{l}x \\
\times\end{array}$ \\
\hline$\times$ & & $\times$ \\
\hline$\times$ & & \\
\hline & $\times$ & \\
\hline$\times$ & & \\
\hline$\dot{x}$ & $\times$ & \\
\hline & . & $\cdot$ \\
\hline$\times$ & . & . \\
\hline . & $x$ & . \\
\hline . & . & $\times$ \\
\hline . & . & $\times$ \\
\hline$\cdot$ & $\cdot$ & $\begin{array}{l}x \\
x\end{array}$ \\
\hline$\dot{x}$ & $:$ & $\hat{.}$ \\
\hline$\times$ & $\dot{0}$ & . \\
\hline$\dot{x}$ & $\begin{array}{l}x \\
x\end{array}$ & : \\
\hline$x$ & . & . \\
\hline$\times$ & $\sigma^{\circ}$ & . \\
\hline$x$ & $x$ & . \\
\hline $\begin{array}{l}\dot{x} \\
x\end{array}$ & : & $\therefore$ \\
\hline$x$ & $\dot{x}$ & . \\
\hline . & $x$ & . \\
\hline
\end{tabular}


TABLE 1 (cont.)

Entomosigma peridinioides J. Schiller Gonyaulax tamarensis Lebour

Gymnodinium achromaticum Lebour

G. albulum Lindeman

G. aureum Kofoid \& Swezy

G. lunula Schütt

G. minutum Lebour

G. puniceum Kofoid \& Swezy

G. splendens Lebour

$G$. variabile Herdman

Gyrodinium pingue (Schütt) Kofoid \&

Swezy

Gyrodinium spp.

Hemidinium nasutum Stein

Katodinium glandula (Herdman) Fott

K. glaucum (Lebour) Fott

Minuscula bipes (Pauls.) Lebour

Oxyrrhis marina Dujardin

Oxytoxum belgicae Meunier

$O$. gracile J. Schiller

Peridiniopsis rotunda Lebour

Peridinium cerasus Pauls.

$P$. depressum Bailey

$P$, diabolus $\mathrm{Cleve}$

$P$. pellucidium (Bergh) Schütt

$P$. trochoideum (Stein) Lemm.

Peridinium spp.

Phalacroma acutum (Schütt) Pavillard

$P$. ovum Schütt

$P$. rotundatum (Clap. \& Lachm.)

Kofoid \& Mich.

Prorocentrum micans Ehrenb.

Protodinium balticum Braarud

Pyrodinium bahamiense Plate

Thecadinium kofoidi Kofoid \& Skog-

bury

Torodinium robustum Kofoid \& Swezy

Warnowia polyphemus (Pouchet) Schiller

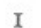

2

BACILLARIOPHYCEAE-36

Amphiprora spp.

Amphora ovalis Kütz

Asterionella japonica Cleve \& Möll.

ex Gran

Bacillaria paxillifer (Müll.) Hendey

Chaetoceros simplex Ostenfeld

C. gracile Schütt

Chaetoceros spp.

Climacosphenia moniligera Ehrenb.

Coscinodiscus centralis Ehrenb.

C. concinnus W. Smith

C. excentricus Ehrenb.

Detonula sp. (Cyclotella nana Hustedt)

Ditylum brightwelli (West) Grun.

Grammatophora marina (Lyng.) Kütz.

Gyrosigma sp.

Leptocylindrus danicus Cleve

Licmophora abbreviata Agardh

Lithodesmium undulatum Ehrenb.

Melosira moniliformis (O.F. Müll.)

Agardh 
TABLE 1 (cont.)

Navicula spp.

$N$. viridis Ehrenb.

Nitzschia closterium (Ehrenb.)

W. Smith

$N$. longissima (de Bréb.) Ralfs ex

Pritch.

N. seriata Cleve

N. sigmoidea (Nitzsch) W. Smith

Paralia sulcata (Ehrenb.) Cleve

Planktoniella sol (Wall) Schütt

Pleurosigma spp.

Rhizosolenia alata Brightw.

$R$. setigera Brightw.

$R$. styliformis Brightw.

Skeletonema costatum (Grev.) Cleve

Skeletonema spp.

Streptotheca tamesis Shrubs.

Thalassionema nitzschioides Hust.

Thalassiosira spp.

II. Zooflagellata-I9

Bicosoeca lacustris J. Clark

Bodo sp. A

Bodo edax Klebs

Bodo sp. B

B. globosus Stein

Bodo sp. C

Bodo sp. D

Bodo sp. E

Bodo spp.

Cercobodo crassicauda (Alexeieff)

Lemmermann

Desmarella moniliformis S. Kent

Helkesimastix faecicola Woodcock \&

Lapage

Monas dangeardii Lemmermann

M. sociabile Meyer

Monosiga ovata S. Kent

Oikomonas termo (Ehrenb.) S. Kent

Pleuromonas jaculans Perty

Rhynchomonas nasuta (Stokes) Klebs Spirochaeta plicatilis Ehrenb.

12. Ciliata-87

Amphisia sp.

Aspidisca costata (Dujardin)

$A$. hexeris Quennerstedt

A. lynceus Ehrenb.

A. pulcherimma Kahl

A. turrita (Ehrenb.)

Chaenea minor Kahl

Chilodonella $\mathrm{sp}$.

C. uncinata (Ehrenb.)

Chilodontopsis caudata Kahl

Chlamydodon triquetrus (O. F. Müll.)

Codonella cratera (Leidy)

Coleps pulcher Spiegel

C. remanei Kahl

Coleps sp.

Condylostoma patens (O. F. Müll.)

Dujardin

Cothurnia plectostyla Stokes

\begin{tabular}{|c|c|c|}
\hline I & 2 & 3 \\
\hline$x$ & $x$ & $x$ \\
\hline . & . & - \\
\hline$x$ & . & $\times$ \\
\hline . & $\times$ & . \\
\hline$\times$ & . & . \\
\hline$\times$ & . & $\times$ \\
\hline$x$ & $x$ & $x$ \\
\hline$x$ & . & . \\
\hline . & $\times$ & . \\
\hline . & $\times$ & \\
\hline$\times$ & . & . \\
\hline$x$ & . & \\
\hline$\times$ & . & $\times$ \\
\hline$\times$ & $\times$ & \\
\hline . & $\times$ & $\times$ \\
\hline . & $\times$ & $\times$ \\
\hline$\times$ & $\times$ & \\
\hline$\times$ & . & . \\
\hline . & . & $x$ \\
\hline$\times$ & . & . \\
\hline . & $\times$ & . \\
\hline . & . & . \\
\hline$\times$ & . & . \\
\hline . & . & . \\
\hline$\times$ & . & . \\
\hline . & . & . \\
\hline . & . & . \\
\hline . & $\times$ & . \\
\hline$\times$ & . & . \\
\hline . & . & . \\
\hline$\times$ & . & \\
\hline$\times$ & . & \\
\hline . & . & . \\
\hline . & . & . \\
\hline$\times$ & . & . \\
\hline . & & \\
\hline
\end{tabular}


TABLE 1 (cont.)

Cristigera media Kahl

C. phoenix Penard

Cyclidium sp. (candens Kahl?)

C. glaucoma O. F. Müll.

Diophrys sp.

D. scutum Dujardin

Dipleurostyla sp.

Dysteria aculeata Clap. \& Lachm.

D. monostyla (Ehrenb.) Stein

Epiclintes ambiguus (Müll.) Bütschli

Enchelyodon farctus Clap. \& Lachm.

Euplotes harpa Stein

$E$. harpa var. marinus Rossolimo

Euplotes sp.

E. vannus (O. F. Müll.)

Favella franciscana Kofoid-Campbell

Frontonia marina Fabre-Dom.

Geleia fossata Kahl

Gruberia uninucleata Kahl

Holosticha discocephalus Kahl

Keronopsis flavicans Kahl

K. multistilata Kahl

Lacrymaria pupula (O. F. Müll.)

Lembus pusillus Quennerstedt

Litonotus cygnus (O. F. Müll.)

L. fasciola (Ehrenb.) Wrzeseniowski Loxophyllum uninucleatum Kahl

Mesodinium cinctum Calkins

M. pulex (Clap. \& Lachm.)

M. rubrum Lohmann

Metacystis truncata Cohn

Nassula aurea Ehrenb.

Opisthostyla sertularium (S. Kent)

Oxytricha pellionella (O. F. Müll.) Ehrenb.

Peritromus californicus Kirby

$P$. emmae Stein

Placus luciae (Kahl)

$P$. striatus Cohn

Pleuronema crassum Dujardin

$P$. marinum Dujardin

Prorodon morgani Kahl

Protocrucia pigerimma (Cohn)

Da Cunha

Remanella margaritifera Kahl

R. rugosa Kahl

Spathidium procerum Kahl

Stenosemella nivalis (Meunier)

Stephanopogon colpoda Entz

$S$. mesnili Lwoff

Strombidium cinctum Kahl

Strombidium $\mathrm{sp}$.

S. lagenula Fauré-Fremiet

S. sulcatum Clap. \& Lachm.

Telostoma ferroi R. \&. L. Grandori

Tiarina fusus (Clap. \& Lachm.)

T. levigata Kofoid-Campbell

Tintinnopsis beroidea Stein emend.

Jörgensen

T. minuta Wailes

$T$. urniger (Entz, Sr.) Daday

Tintinnopsis spp. unid.

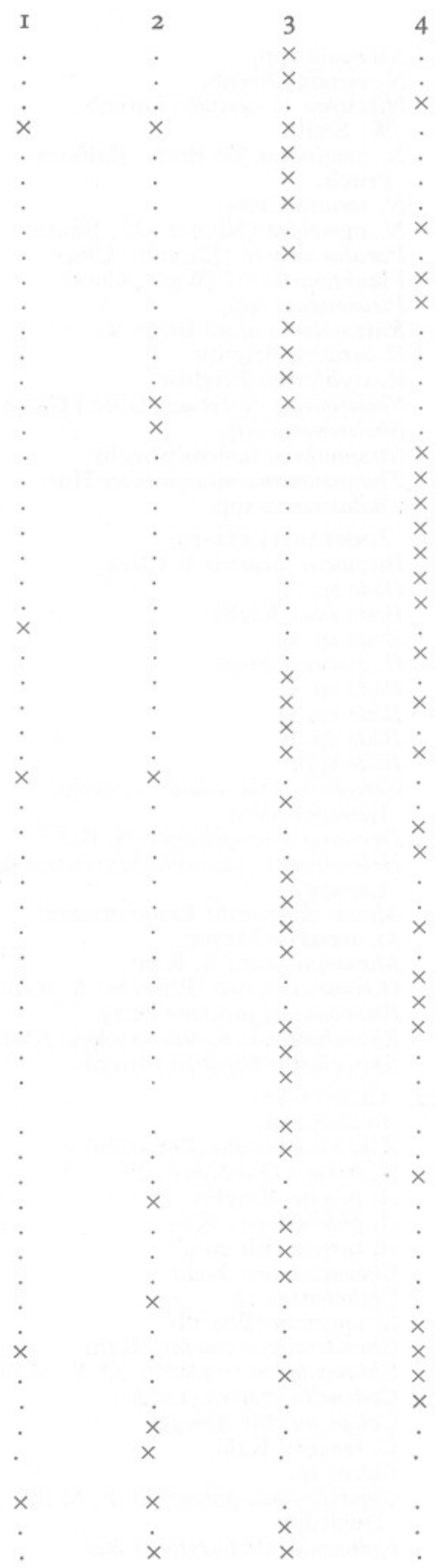


TABLE 1 (cont.)

Trachelocerca sp.

T. entzi Kahl

T. phoenicopterus Cohn

Trachelostyla pediculiformis (Cohn)

Trichopelma torpens $\mathrm{Kahl}$

Trochilia fluviatilis Smith

T. salina Entz

Uronema filificum $\mathrm{Kahl}$

U. marinum Dujardin

Uronychia setigera Calkins

U. transfuga (O. F. Müll.)

Urostrongylum caudatum Kahl

Vaginicola doliolum Penard

Vorticellidae, unid.

$\begin{array}{cccc}\text { I. } & 2 & 3 & 4 \\ . & . & \times & \dot{ } \\ . & i & \times & . \\ . & . & \times & . \\ . & . & \times & . \\ . & . & \times & \times \\ . & \times & \times & . \\ . & . & \times & . \\ . & . & \times & . \\ . & . & \times & \times \\ . & . & \times & \times\end{array}$

\section{QUANTITATIVE RESULTS}

Comparison of the sand and sediment population with that of the plankton indicates that the latter is poor in number of species, the former rich. This statement applies even if the blue-green algae, bacteria and rhizopods are included. These, except for the sulphur bacteria, are omitted from this consideration because identification of many was impossible. Biomass may be another matter, because the sand and the sediment interfaces are thin layers in which bacteria, colourless euglenids and ciliates are concentrated, whereas the plankton Chrysophyceae, diatoms and dinoflagellates tend to be distributed through a considerable water column. No estimates of the total sediment and sand populations were made, but on two occasions the population of plankton per ml. was counted. The numbers are large, as shown by Table 2, which is a count obtained by centrifuging water freshly obtained from beyond the breakwater. However, $1267 / \mathrm{ml}$. is not a large population in comparison with bacterial populations, or blooms of Eutreptia, or Gymnodinium breve. The I 7 largest organisms in Table 2 account for only $60 \cdot 75$ individuals per ml. Of the others many are but $\mathrm{I} \cdot 5-5 \cdot 0 \mu$ in length, and virtually impossible to identify with ordinary light microscopy. Probably a large number belong to such organisms as Micromonas pusilla whose status is in doubt (Manton, I959; Manton \& Parke, I960), Chrysochromulina strobilus (Parke, Manton \& Clarke, I959), and others of similar size. The large group of 'Cells, incertae sedis' cannot even be called colourless or coloured except under the oil-immersion objective; and it seems probable that accurate counting and identification can be done only on fixed and stained material.

Table 2 is intended only to give an indication that a sizeable plankton population may be expected, at least during the summer. There is little doubt that huge numbers of very small non-bacterial cells are quite important ecologically. Such has been the experience of the oyster industry with Nannochloris bacillaris in Great South Bay, New York (Lackey, Vanderborg \& 
Glancy, I949); and with Detonula sp. (= Cyclotella nana Hustedt: see Guillard \& Ryther, 1962).

TABLE 2. ORGANISMS PER ML. OF RAW WATER, FROM THE REGION OUTSIDE THE BREAKWATER AT PLYMOUTH, 20 AUGUST 1962

\begin{tabular}{|c|c|c|c|}
\hline $\begin{array}{l}\text { Chlorophyceae, unid. } \\
\text { Volvocales }\end{array}$ & 54.00 & $\begin{array}{l}\text { Phalacroma rotundatum } \\
\text { Minuscula bipes }\end{array}$ & $\begin{array}{l}3.00 \\
0.25\end{array}$ \\
\hline Pyramimonas grossi & 3.00 & Protodinium balticum & 6.00 \\
\hline Chrysophyceae & & Torodinium robustum & 0.25 \\
\hline Chryschromulina, unid. & 123.00 & Zooflagellata & \\
\hline Kephyrion ovum & 3.00 & Monosiga ovata & \\
\hline Chrysophyceae, unid. & $327 \cdot 00$ & Monads, various & $156 \cdot 0$ \\
\hline Cryptophyceae & & Cells incertae sedis & $330 \cdot \mathrm{C}$ \\
\hline Chilomonas marina & 0.75 & Bacillarieae & \\
\hline Rhodomonas spp. & $108 \cdot 00$ & Asterionella japonica & \\
\hline Coccolithophora & & Detonula sp. & $45 \cdot 00$ \\
\hline Pontosphaera sp. & $45^{\circ} 00$ & Paralia sulcata & \\
\hline $\begin{array}{l}\text { Syracosphaera sp. } \\
\text { Silicoflagellata }\end{array}$ & 3.00 & $\begin{array}{l}\text { Navicula spp. } \\
\text { Nitzschia closterium }\end{array}$ & $\begin{array}{r}3.00 \\
36.00\end{array}$ \\
\hline Dictyocha fibula & 0.25 & Thalassiosira $\mathrm{sp}$. & 0.75 \\
\hline Dinoflagellata & & Ciliata & \\
\hline Gymnodinium spp. & $\mathrm{I} \cdot 50$ & Mesodinium rubrum & \\
\hline G. albulum & $I \cdot 25$ & Uronema marinum & 0.25 \\
\hline Gyrodinium sp. & $I \cdot 50$ & & \\
\hline $\begin{array}{l}\text { G. pingue } \\
\text { Katodinium glaucum }\end{array}$ & $\begin{array}{l}0.50 \\
0.25\end{array}$ & & $1267 \cdot 0$ \\
\hline
\end{tabular}

Our intent at Plymouth was to ascertain the kinds and distribution of the colourless euglenids. Four distinct habitats were studied: open water, sediment interfaces in the Plym and Tamar rivers, and the sand between high and low tide. The Plym flats with their clay deposits and large numbers of sulphur bacteria, denoting considerable $\mathrm{H}_{2} \mathrm{~S}$ and organic deposits, were expected to have low populations in both number and kind. Actually they were highest in both categories. The Tamar, on the contrary, with a very large attached seaweed population, had low numbers of microbiotic types, wherever sampled. Its waters, however, contained many Gonyaulax tamarensis. The differences between the habitat types are clearly indicated by sulphur bacteria, saprophytic flagellates, and a relatively small ciliate group. The harbour sand has a large ciliate group, a number of saprophytic flagellates including the recently described Pentamonas spinifera Lackey, I96I; and certain sand-dwelling dinoflagellates. Because of organic matter entering the harbour, larger numbers were expected than were found. The same statement applies to the plankton. Evidently the high rise and fall of the tides affords ample dilution so that fertilization in the harbour area is low. While blackening of the mud and some slight odour of $\mathrm{H}_{2} \mathrm{~S}$ in the Plym estuary indicates active decomposition of organic matter there, neither loss of $\mathrm{O}_{2}$ nor accumulation of $\mathrm{H}_{2} \mathrm{~S}$ become limiting factors. Very few anaerobic organisms were found there.

Most of the organisms have been found in tidal waters elsewhere. Some are members of well recognized associations, and a few, denoted in Table I by the use of letters as a provisional designation, have not been sufficiently 
studied for taxonomic inclusion. Though as yet without a name, they are indicated in a special way because they occur in sufficient numbers in this and other places to be available for taxonomic study.

\section{REFERENCES}

Guillard, Robert R. L. \& Ryther, John H., I962. Studies of marine planktonic Diatoms. I Cyclotella nana Hustedt, and Detonula confervacea (Cleve) Gran. Canad. F. Microbiol., Vol. 8, pp. 229-239.

LACKEY, JAMES B., 1961. Three new colorless Euglenophyceae from marine situations. Arch. Mikrobiol., Vol. 42, pp. 190-195.

Lackey, James B., VANDeRborgh, George, Jr. \& Glancy, Joseph B., I949. Plankton of waters overlying shellfish grounds. Proc. nat. Shell-fish. Ass., 1949, pp. I-5.

MANTON, IRENE, 1959. Electron microscopical observations on a very small flagellate: the problem of Chromulina pusilla Butcher. f. mar. biol. Ass. U.K., Vol. 38, pp. 319-33.

MANTON, I. \& PARKE, M., 1960. Further observations on small green flagellates with special reference to possible relatives of Chromulina pustlla Butcher. F. mar. biol. Ass. U.K., Vol. 39, pp. 275-298.

Marine Biological Association, 1957. Plymouth Marine Fauna. 3rd Edition.

PARKe, M., MANTon, I. \& Clarke, B., I959. Studies on marine flagellates. V. Morphology and microanatomy of Chrysochromulina strobilus sp.nov. 7. mar. biol. Ass. U.K., Vol. 38, pp. 169-88. 